\title{
Multiple-Filtering Process and Its Application in Edge Detection
}

\author{
Jing Li and Chunyan Wang \\ Department of Electrical and Computer Engineering, Concordia University \\ 1455 de Maisonneuve Blvd. West Montreal, Quebec, Canada H3G 1M8 \\ $\{1$ jin9, chunyan\}@ece.concordia.ca
}

\begin{abstract}
In this paper, a procedure of edge detection for a high dynamic range image with damaged edge information is proposed. This procedure is based on a scheme of multiple filtering processes which does not include any segmentation of the image. Three different filtering processes are designed to generate three gradient maps, in each of which gradients are calculated and modulated by using a specific filter. The enhanced gradients, i.e. those modulated correctly, are identified in each of the three gradient maps by using a selection algorithm. They are taken to generate a complete edge map. This procedure allows varieties of edge gradient enhancements applied in the same image by employing a set of simple filters without segmentation. The effectiveness of the detection process has been confirmed by simulations.
\end{abstract}

Index Terms - multiple-filtering, edge detection, high dynamic range image, gradient magnitude based detection

\section{Introduction}

In many image filtering tasks, the images to be processed are usually acquired under non-ideal conditions, some of which can lead to complex signal degradation in the image. One of such examples is high dynamic range (HDR) images captured by an ordinary camera with some details damaged. A complex filtering process can be used to cope with the various intensity variances. One of such examples is found in [1]. However, to reduce the computation complexity, a multiple-process structure can be considered. Each of the processes is to be made to enhance the edge signals under one condition [2]. In this case, one needs to identify the regions in order to apply appropriate processes to these regions, respectively. For this purpose, segmenting an image into regions for different filtering processes is an approach [3]. However, identifying the real boundaries of the regions may not be an easy task. To avoid this problem, one would consider an approach without explicit image segmentation. In case of processing HDR images, multiple exposures can be used to generate several images. Detailed information is captured in a series of images, and a fusion algorithm is developed to merge them [4] [5].

In this paper, we propose a method to detect edge signals effectively in the HDR images based on the scheme of segmentation-free multiple filtering processes.

\section{Design of multiple filtering processes and selection algorithm}

As mentioned previously, an image acquired under complex condition may need multiple processes. We can use multiple filters to generate multiple filtered images and then to identify the regions, or the pixels in these regions, resulting from the most suitable process. Thus, the identification of the regions is carried out after the filtering processes, while the segmentation is spared. In this section, the scheme of the process is described, and a procedure of edge detection is designed by applying the scheme.

\subsection{Scheme of the multiple filtering processes}

The segmentation-free multiple filtering processes is described in this sub-section. Assume that, to process an image acquired under complex conditions, $n$ simple filtering processes are designed so that each of them targets one of the conditions. Each filtering process is applied to the entire original image $(O I)$, and $n$ filtered images (FIs) are then generated. A selection algorithm is designed and applied to these FIs to produce a good filtered image. To the end, one needs to look into the two image levels, the regions and the pixels.

Considering the region level, each filtering process is designed to suit the regions generated under the matched condition. Therefore, in each FI, only the pixels in these regions, considered as "matched regions", can receive the correct results from this filtering process, and the rest of the FI are the "unmatched regions". The selection algorithm can be designed to distinguish the matched regions from unmatched ones. In this way, "unmatched regions" will be filtered out by the selection process from all the FIs, and only the "matched regions" are preserved to generate a complete filtered image.

Considering the pixel level, at each pixel position, $n$ values resulting from $n$ filtering processes are received. However, only one of them is the "good result" produced by the most appropriate filtering process. With these $n$ results, all the scenarios generated by the $n$ processes need to be studied. Based on the analysis of the possible scenarios, the selection criteria are established and they can be described by a mathematic model which is used to develop the selection algorithm to identify the desirable result from the $n$ values. The selection is processed in the pixel level, and the 
boundaries of all the regions are defined automatically pixel by pixel. It should be mentioned that this identification of the boundaries is done in the filtered domain, which can be easier than that is in the original spatial domain.

In the following sub-section, the design of an edge detection procedure is proposed while applying the scheme.

\subsection{Design of the edge detection procedure}

With the scheme described above, a procedure is designed for an edge detection to be applied to an HDR image. Due to the nonlinear characteristics of image sensor, the edge signals in different locations may be attenuated in a complex manner related to the intensity variation. In this case, the gradients produced by the edges and those by the noise may not be easily distinguished, and a fixed threshold or filtering process with fixed parameters is not suitable. To extract all the edge signals, a group of filtering processes need to be designed, and each of them is to match one of the intensity conditions. The procedure of this edge detection is shown in Fig. 1. A group of filtered images (FIs) are generated by the filtering processes. In this design, each of the FIs is a gradient magnitude image with enhanced gradients in certain regions. Like most of the edge detections, the edge map is obtained by binarizing the gradient signal at each pixel position with a threshold value. In this procedure, since multiple gradients are produced at each pixel, we need a selection process to sort the gradient data for the binarization.

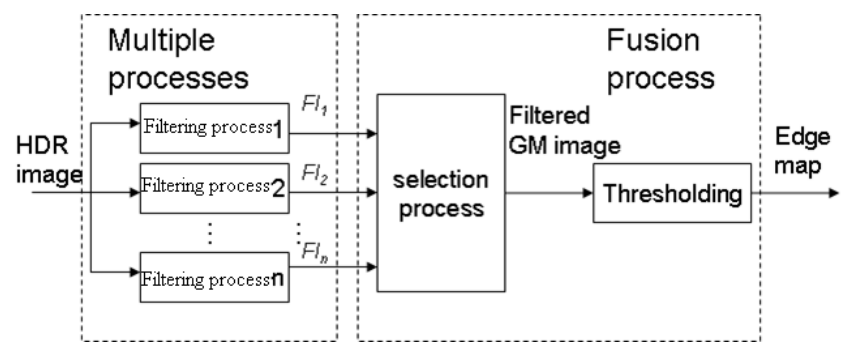

Fig. 1. Procedure of the edge detection.

\subsubsection{Design of filtering processes}

The signal of an HDR image can vary over a very wide range, e.g. 5 decades or more, which greatly exceeds the range of linear operation of most of the image sensors. Instead of using multiple exposures to acquiring all the detail information in different ranges [5], the proposed procedure is designed to extract the information from an image acquired or transferred by range-limited devices. To enhance or restore the edge gradients, different degrees of signal attenuation in the regions under different intensity conditions need to be investigated.

As mentioned previously, the signal attenuation during the image acquisition is due to the nonlinear characteristics of the sensors. In case of bright illumination, the intensity differences are attenuated because of the saturation of the sensor. Under the condition of weak illumination, visual signal loss is mainly caused by the under exposure. In the first case, signal gradient decreases while the intensity is increasing and that in the other case increases with the intensity. Therefore, the gain applied in the gradient process should be different in the two cases.

In this design, the intensity range is divided into three sub-ranges, corresponding to dark, median and bright regions for simplicity of the procedure. The filtering process is, in fact, a modulated gradient calculation process. Let $G$ denote the gradient magnitude at a given pixel position, and it is obtained by applying a set of gradient kernels, such as that of Sobel. However, the parameter of the filtering process needs to be related to the local intensity conditions, and should be different from region to region.

(1) In a dark region, the under exposure makes edge gradients weaker in a weaker background. If $G_{D}$ denotes the result of the filtering process in this region, it is defined as $G_{D}=\frac{G}{\sum \sqrt{I_{i}}}$, where $\sum \sqrt{I_{i}}$ is the sum of the square roots of the original pixel signals in the neighborhood;

(2) In a bright region, due to the device saturation, the signals are more attenuated if the background level is higher. Therefore, the filtering result in this case is given by $G_{B}=G \cdot \sum I_{i}$;

(3) There must be regions, called median regions, in which signals are acquired under the illumination condition between the two cases discussed above. Let $G_{M}$ be the result of the filtering process in this case, we have $G_{M}=G$.

The filtering processes described above are all based on gradient calculation, and the gradient values are modulated by different filtering processes. The factor of the modulation is variable depending on the original pixel signals in the pixel location. In general, which kind of regions, dark, median or bright, the pixel is located in, determines which of the three different filters is the matched one. Please note that in the stage of the filtering process, we do not need to identify which pixels should be processed by its matched filtering process, as every pixel is filtered three times without discrimination. But each of the three filters produces the gradients in a discriminative manner according to the original pixel signals in the neighborhood. The following sub-section is dedicated to the description of the selection algorithm to differentiate, in each of the three NFI, the pixels in the matched regions from those in the unmatched ones.

\subsubsection{Design of the selection algorithm}

The three filtering processes result in, at each pixel position, three modulated gradient signals. The selection algorithm is needed to identify the one that is generated by the most appropriate filtering process among the three. The results of this selection are then used to determine the threshold of the selected regions to produce the final results. 
As each of the filtering processes is designed to enhance the edge gradients in certain regions, different filters have different signal-dependent parameters. The actual gains of the processes are different from one another. Thus, the results generated from these processes need to be normalized with a signal-and-process-dependent parameter in order to implement the selection process. In this design, the pixels of each $F I$ are normalized by the maximum pixel value of the $F I$. The normalized FIs are called NFIs.

Let the normalized $G_{D}, G_{B}$ and $G_{M}$ be $G_{N D}, G_{N B}$ and $G_{N M}$ respectively. At a given pixel position $(i, j)$ in each of the NFIs, the normalized values of the processes are given by the follow equations:

$$
\begin{aligned}
& G_{N D}(i, j)=G_{D}(i, j) / \operatorname{Max}\left\{G_{D}\right\}=\frac{G(i, j)}{\sum \sqrt{I(i, j)}} / \operatorname{Max}\left\{G_{D}\right\} \\
& G_{N M}(i, j)=G_{M}(i, j) / \operatorname{Max}\left\{G_{N}\right\}=G(i, j) / \operatorname{Max}\left\{G_{M}\right\} \\
& G_{N B}(i, j)=G_{B}(i, j) / \operatorname{Max}\left\{G_{B}\right\}=G(i, j) \cdot \sum I(i, j) / \operatorname{Max}\left\{G_{B}\right\}
\end{aligned}
$$

The above equations show that $G_{N D}(i, j), G_{N B}(i, j)$ and $G_{N M}(i, j)$ are determined by the gradient value $G(i, j)$ and the original pixel signals in the neighborhood. For a given $G$, the characteristics of $G_{N D}, G_{N B}$ and $G_{N M}$ versus the original pixel signal $I(i, j)$ are shown in Fig. 2. If the original pixel signal is very weak, produced by a low intensity, among the three normalized filtering results, $G_{N D}$ should be the correct one to be selected. Similarly, in case that $I(i, j)$ is produced by a high intensity, $G_{N B}$ needs to be identified as the right result. The plots in Fig. 2 show that, no matter the level of the original signal $I(i, j)$, the largest value of $G_{N D}, G_{N B}$ and $G_{N M}$, is the one that suits $I(i, j)$. Thus, the computation for the selection is to calculate $G_{N D}, G_{N B}$ and $G_{N M}$ and the maximum value of the three is identified as the "good" one. Once the pixel selection is done, all the "good" results generated from the three processes make a complete normalized filtered image. The following step is to perform binarization in the selected pixels in this complete image.

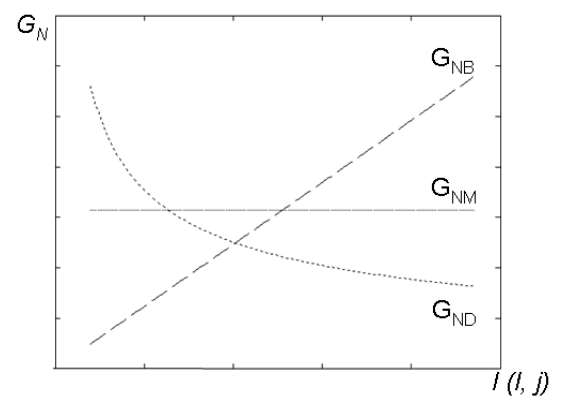

Fig. 2. Normalized results generated by the three filtering processes versus the level of the original signal with a given $G$ at a given position $(i, j)$. The dotted curve is of $G_{N D}$; the dashed one of $G_{N B}$; the solid one of $G_{N M}$.

As the pixel signals are generated by the three different filtering processes, three threshold values are applied for the binarization. Each of them is determined by the average value of the selected pixel signals from the same NFI, and is then applied to these pixels in the binarization. The completed edge map is then generated.

The proposed procedure of the edge detection is implemented in a computation diagram shown in Fig. 3, and it proceeds as follows.

(1) The original HDR image receives a preprocess with a Gaussian smooth filtering;

(2) Three FIs are generated by using a set of classical Sobel operators with the different modulation specified in Sub-Section 2.2.1;

(3) The pixels in each $F I$ are normalized to generate the normalized $F I$ images $N F I_{D}, N F I_{M}$ and $N F I_{B}$;

(4) At every pixel, the local result is compared to the other two, from the other NFIs respectively. For each NFI, if the local one is the maximum among the three, it will be kept at this position; otherwise, the pixel value at this position will be replaced by zero. The outputs of this stage are the images $S I_{D}, S I_{M}$ and $S I_{B}$, with $S I$ s standing for the selected images.

(5) In each of the SIs, the threshold value is determined by the average value of all the non-zero pixels and applied to this $S I$ to perform binarization. After a thinning process, three edge maps $E M_{D}, E M_{M}$ and $E M_{B}$ are then generated and merged.

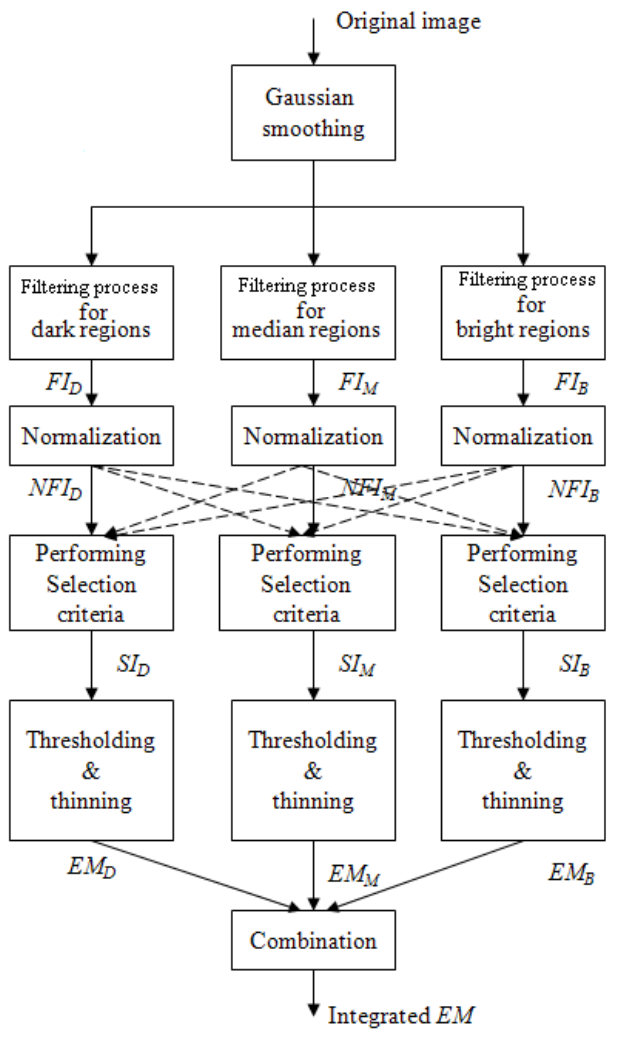

Fig. 3. Computation diagram of the edge detection procedure. 


\section{Simulation results and evaluation}

To evaluate its effectiveness and quality, the proposed edge detection method has been simulated with some images of HDR scenes. The simulation has two parts for the subject observations and objective measurements, respectively.

As the original images are obtained by a single exposure, some regions suffer under-exposure and some others over-exposure. The results of one of the cases are shown in Fig.4. The three edge maps are obtained by applying the different methods, namely simple SOBEL, Canny and the proposed one, respectively. It is easily observed that by using the proposed method, much more edges can be detected correctly. The simulation results of the other HDR images have confirmed the same.

For the objective measurement, Pratt's Figure of Merit (PFOM) has been used [7]. It is calculated by comparing the edge map generated by using the proposed method with an "ideal" edge map of the same scene. If the two edge maps are identical, the PFOM will be equal to unity. In this simulation, the "ideal" edge map is produced from a visualized HDR image of the same scene illustrated in Fig.5 (a). This image is acquired by multiple-exposure, so that good contrast in all the irradiance ranges is preserved. The "ideal" edge map used as the reference shown in Fig.5 (b) is obtained by applying Canny edge detector in the visualized HDR image. The results of the PFOM are displayed in Table 1, which is demonstrated qualitatively that the edge map produced by the proposed method has significantly more likeness to the "ideal" one than those by the two other detections.

It should be noted that, in the original image illustrated in Fig.4 (a), the contrast information over a wide intensity range is severely damaged and the proposed method is particularly effective to extract the edge signals in this kind of images.

Table.1 PFOM values obtained by the three methods

\begin{tabular}{|c|c|c|}
\hline Sobel & Canny & Proposed method \\
\hline 0.6663 & 0.7058 & $\mathbf{0 . 8 4 8 2}$ \\
\hline
\end{tabular}

\section{Conclusion}

In this paper, we first discussed a scheme of multiple filtering processes. Instead of segmenting an image into regions for different processes, it is done by applying each of the processes to the original image to generate multiple filtered images and a selection algorithm is used in each filtered image to identify the pixel signals resulting from the most appropriate process. A procedure of edge detection for HDR images has been proposed by using this scheme. In this procedure, the gradient signals are modulated in a discriminative manner according to the features of signal deformation. This discriminative modulation is implemented by three simple filters to generate three filtered images. A simple selection algorithm is applied to produce the edge map containing detail information of the original image in all regions, even though a lot of it has been damaged in the original one. The evaluation results including subjective observations and objective measurements show that the proposed method can effectively detect edge signals in HDR images.

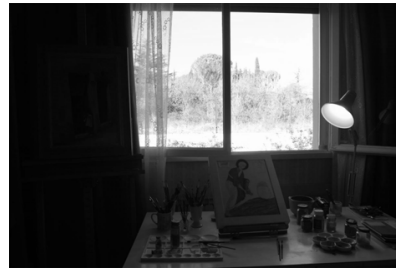

(a) original image [6]

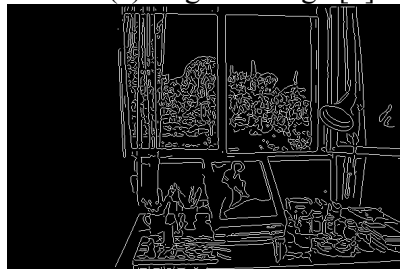

(c) Canny

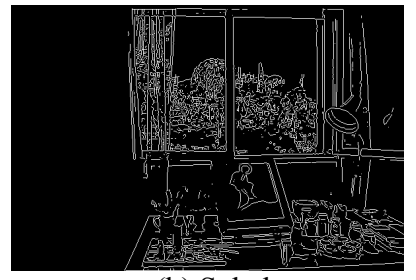

(b) Sobel

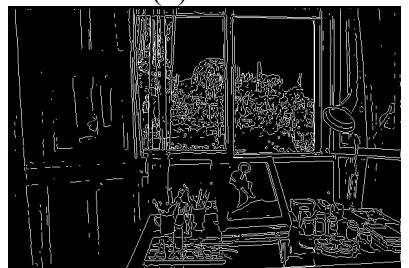

(d) proposed method
Fig. 4. Original image and the edge maps obtained by using different filtering processes.

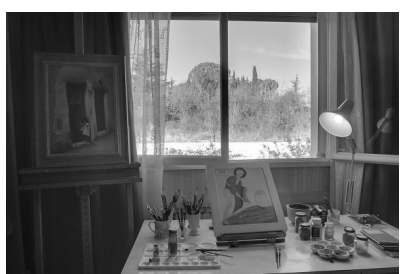

(a) visualized HDR image[6]

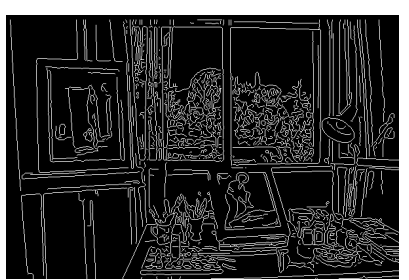

(b) reference edge map
Fig. 5 HDR visualized image and the reference edge map.

\section{References}

[1] E.J. Wharton, K. Panetta, S.S. Agaian, "Logarithmic edge detection with applications", Jour. of Computers, Vol. 3, Issue 9, pp. 3346-3351, September 2008.

[2] R. J. Qian, T. S. Huang, "optimal Edge Detection in Two-Dimensional Image," IEEE Trans. of Image Processing, Vol. 5, pp. 1215 - 1220, 1996.

[3] W.K. Pratt, J. Wiley \& Sons, Digital image processing: PIKS Scientific inside $4^{\text {th }} e d, 2007$.

[4] W. Kao, "High Dynamic Range Imaging by Fusing Multiple Raw Images and Tone Reproduction," IEEE Trans. of Consumer Electronics, Volume 54, Issue 1, pp. 10 - 15, February 2008.

[5] A.R. Varkonyi-Koczy, A. Rovid, T. Hashimoto, "Gradient-Based Synthesized Multiple Exposure Time Color HDR Image," IEEE Trans. of Instrumentation and Measurement, Vol. 57, Issue 8, pp. 1779-1785, August 2008.

[6] Gallery of HDR photos http://www.hdrsoft.com/index.html.

[7] W. K. Pratt, Digital Image Processing, 1991. 